\title{
Proposed Algorithm for Surveillance Applications
}

\author{
Youssef Benabbassi \\ University of Bechar \\ RIIR Laboratory, Oran \\ Bechar, Algeria
}

\author{
Hafid Haffaf \\ University of Oran \\ RIIR Laboratory, Oran \\ Oran, Algeria
}

\author{
Congduc Pham \\ University of Pau \\ Pau LIUPPA, France \\ Pau, France
}

\begin{abstract}
Technological systems are vulnerable to faults. In many fault situations, the system operation has to be stopped to avoid damage to machinery and humans. As a consequence, the detection and the handling of faults play an increasing role in modern technology, where many highly automated components interact in a complex way such that a fault in a single component may cause the malfunction of the whole system. This work introduces the main ideas of fault diagnosis and fault-tolerant control under the optics of various research work done in this area. It presents the Arduino technology in both hardware and software sides. The purpose of this paper is to propose a diagnostic algorithm based on this technology. A case study is proposed for this setting. Moreover, we explained and discussed the result of our algorithm.
\end{abstract}

Keywords: Technological systems, control system, actuator faults, Arduino, sensors faults

\section{INTRODUCTION}

The manufacturing systems consist of many different machine tools, robots and transportation systems all of which have to correctly satisfy their purpose in order to ensure an efficient and high-quality production. Mobile communication provides another example where networked components interact so heavily that component faults have far reaching consequences.

In the general sense, a fault is something that changes the behavior of a system such that the system does no longer satisfy its purpose. In large systems, every component has been designed to accomplish a certain function and the overall system works satisfactorily only if all components provide the service they are designed for. Therefore, a fault in a single component usually changes the performance of the overall system. The presence of a fault detection and isolation (FDI) system is then necessary to detect the occurrence of a fault and isolate it. Normally, the task which comes after the isolation of a fault is its repairing. The problem of FDI and Fault Tolerant Control (FTC) is an important problem to deal with, since faults in sensors, actuators and components are usually associated to increasing operating costs, offspecification production, line shut-down and possible detrimental environment impact.

Overall, fault-tolerant control is a complex interdisciplinary research field that covers a diverse range of engineering disciplines, such as modeling and identification, applied mathematics, applied statistics, stochastic system theory, reliability and risk analysis, computing, communication, control, signal processing, sensors and actuators, as well as hardware and software implementation techniques.

The measures should be carried out by the control equipment, in order to avoid production deteriorations or damage to machines and humans. Their aim is to make the system fault tolerant. The control algorithm adapts to the faulty plant and the overall system satisfies its function again as it is shown in Figure 1

In the literature, most of the motivation and research work in fault tolerant control involves solving problems encountered in safety critical systems such as aircraft.
In [1] the author proposes a solution for fault detection and isolation using system dynamics identification techniques. In [2] the authors propose a fault detection and isolation scheme for industrial systems based on multiple operating model. Rosa and al. in [3] describe an application of a new fault detection and isolation (FDI) technique based on set-valued observers (SVOs) to a linear parameter varying (LPV) longitudinal aircraft dynamic model. The authors in [4] propose parameter estimation methods for fault detection and isolation. In [5] Tharrault and al. propose fault detection and isolation with robust principal component analysis. This proposed scheme avoids the combinatorial explosion of faulty scenarios related to multiple faults to be considered. The authors in [6] designed closed-loop fault-tolerant control for uncertain nonlinear systems. This solution is based on a new algebraic estimation technique of the derivatives of a time signal. This yields good estimates of the unknown parameters and of the residuals of the fault indicators.

A general active fault-tolerant control framework is proposed in [7] for nonlinear systems with sensor faults. According to their identifiability, all sensor faults are divided into two classes: identifiable faults and non-identifiable faults.

In [8] the authors propose a new fault-tolerant control methodology using adaptive estimation and control approaches based on the learning capabilities of neural networks or fuzzy systems. On-line approximation-based stable adaptive neural/fuzzy control is studied for a class of input-output feedback linearizable time-varying nonlinear systems. The authors in [9] discussed the problem of designing fault tolerant compensators that stabilize a given system both in the nominal situation, as well as in the situation where one of the sensors or one of the actuators has failed.

The paper is organized as follows. Section 2 presents the Fault-Tolerant Control. Section 3 presents a case study. Section 4 presents our algorithm proposed for diagnosis based on Aduino programming. Finally, section 5 concludes the paper and points out open research problems. 


\section{FAULT-TOLERANT CONTROL}

\subsection{Definitions}

System: A system is a set of interconnected components. Each of the components has been chosen or designed by the system engineer so as to achieve some function of interest. A function describes what the design engineer expects the components to perform, independently of how it is performed. A component performs some function because it has been designed so as to exploit some physical principles. Which in general are expressed by some relationships between the time evolution of some system variables. Such relationships are called constraints, and the time evolution of a variable is called its trajectory [10][11].

Fault: A fault in a dynamical system is a deviation of the system structure or the system parameters from the nominal situation. Examples for structural changes are the blocking of an actuator, the loss of a sensor or the disconnection of a system component.

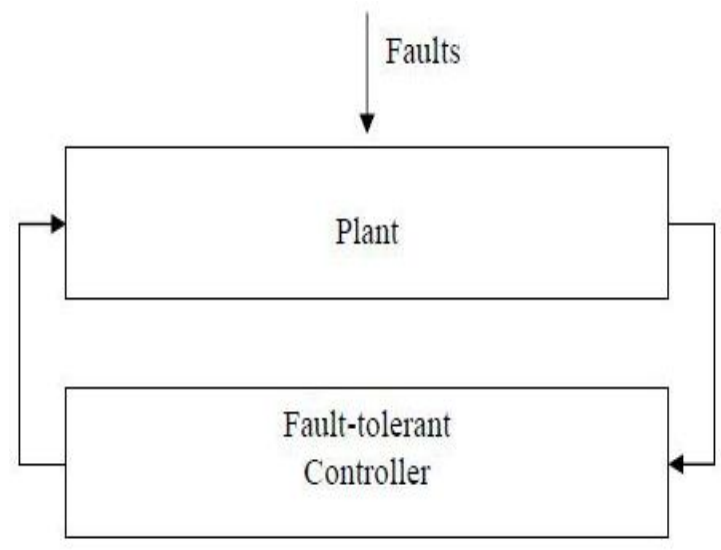

Figure 1. Fault-tolerant system

\subsection{Classification of faults}

The faults are often classified as follows:

Plant faults: such faults change the dynamical I/O properties of the system.

Sensor fault: the plant properties are not affected, but the sensor readings have substantial errors.

Actuators faults: the plant properties are not affected, but the influence of the controller on the plant is interrupted or modified.

\subsection{Fault-tolerant control notions}

The aims of fault-tolerant control are related to these notions, which result from different views on faulty systems:

\subsubsection{Safety}

It describes the absence of danger. A safety system is a part of the control equipment that protects a technological system from permanent damage. A controlled shut-down brings the technological process into a safe state, if specified conditions are met. Dedicated actuators stop the process. The over-all system is then called a fail-safe system.

\subsubsection{Reliability}

It is the probability that a system accomplishes its intended function for a specified period of time under normal conditions. Fault-tolerant control cannot change the reliability of the plant components, but it improves the reliability of the overall system, because with a fault-tolerant controller the overall system remains operational after the appearance of faults.

\subsubsection{Availability}

It is the probability of a system to be operational when needed. Contrary to reliability it also depends on the maintenance policies, which are applied to the system components.

\subsubsection{Dependability}

It lumps together the three properties of reliability, availability and safety. A dependable system is a fail-safe system with high availability and reliability.

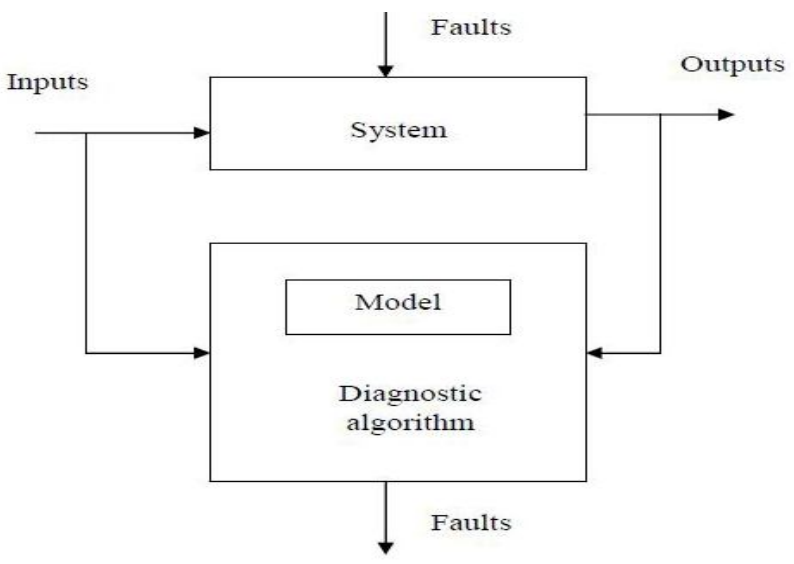

Figure 2. Fault diagnosis

\subsection{Physical redundancy and analytical redundancy}

Fault tolerance necessitates redundancies. The main advantage of fault-tolerant control over measures for fault tolerance is the fact that fault-tolerant control makes intelligent use of the redundancies included in the system and in the information about the system in order to increase the system availability. The analytical redundancy is cheaper than duplicating all vulnerable components.

Three methods for ensuring fault tolerance are:

\subsubsection{Robust control}

A fixed controller is designed that tolerates changes of the plant dynamics. The controlled system satisfies its goals under all faulty conditions. Fault tolerance is obtained without changing the controller parameters. It is, therefore, called passive fault tolerance. 


\subsubsection{Adaptive control}

The controller parameters are adapted to changes of the plant parameters. If these changes are caused by some fault, adaptive control may provide active fault tolerance.

\subsubsection{Diagnosis steps}

For fault-tolerant control, the location and the magnitude of the fault have to be found. Different names are used to distinguish the diagnosis steps according to their depth:

- Fault detection: Decide whether or not a fault has occurred. This step determines the time at which the system is subject to some fault.

- Fault isolation: find in which component a fault has occurred. This step determines the location of the fault.

- Fault identification and fault estimation: identify the fault and estimate its magnitude. This step determines the kind of fault and its severity.

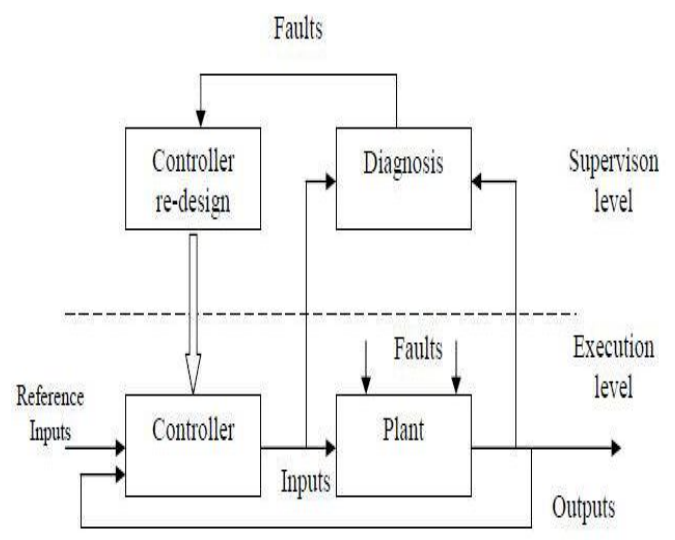

Figure 3. Architecture of fault-tolerant control

To diagnose a system by testing the consistency of the measurements with a model is a general idea, which does not depend on the kind of mode used.

Several direct consequences of this principle should be mentioned:

- Fault detection is possible without any information about the behavior of the faulty plant. Fault detection algorithms use only a model of the nominal plant. The main idea is to identify deviations of the current system behavior from the nominal behavior, which is possible without a list of all possible faults.

- Without information about the faults and about the way in which the faults affect the system, no fault isolation and identification is possible. In order to identify the fault, fault models have to be known.

- With a given measurement configuration, not all faults can be distinguished. Diagnosability considerations can be used to determine those faults that can be separately identified. Generally, the way to make a system fault-tolerant consists of two steps:
Fault diagnosis: The existence of faults has to be detected and the faults have to be identified.

Control re-designs: The controller has to be adapted to the faulty situation so that the overall system continues to satisfy its goal.

\section{CASE STUDY}

This section presents the technique to monitor our process using an Arduino-based microcontroller programmed. More details on the Arduino-Uno and programming will be given in the next section. Our process is seen as a functional system which outputs values indicating the status of its operation. This operating state is classified either correct mode operation, malfunction or faulty. The first output, indicates the temperature $(\mathrm{T})$ released, and the second indicates the pressure $(\mathrm{P})$ collected during operation of the process. In nominal mode, the values of these two variables are the zero state (0). So the system is safe mode. But once one of the two values of these two variables changes to state one (1) this means that the system switches to malfunction. An alarm is triggered, indicating the presence of this fault, which requires adequate operation, either cooler level, or at the compressor. In the worst case we ordered a forced shutdown of the system. As these two variables are monitored, one can have the four possible modes of operation of the system, they are given as follows in table 1:

\begin{tabular}{|c|c|c|}
\hline $\begin{array}{c}\text { Temperature } \\
\text { (T) }\end{array}$ & $\begin{array}{c}\text { Pressure } \\
(\mathbf{P})\end{array}$ & Operating Mode \\
\hline 0 & 0 & Functional (safe) \\
\hline 0 & 1 & Dysfunction (in compressor) \\
\hline 1 & 0 & Dysfunction (in cooling) \\
\hline 1 & 1 & Failure \\
\hline
\end{tabular}

Table 1. Operating modes of the process

Our process monitoring scheme based on an Arduino microcontroller is given as follows in Figure 4:

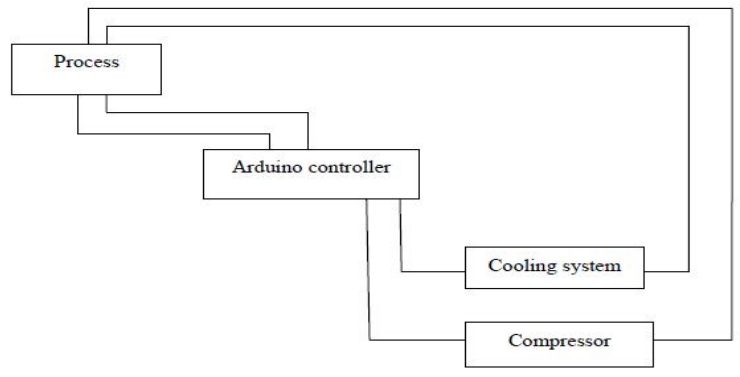

Figure 4. Process monitoring scheme based on Arduino

\section{PROGRAMMING ARDUINO}

The Arduino is a microcontroller board based on a (minicomputer) Atmel ATMEGA8 or ATMEGA168. It has in its basic version $1 \mathrm{~KB}$ of RAM and $8 \mathrm{~K}$ of flash memory for storing programs. It can be connected to 13 digital inputs or outputs, 3 PWM (up to 3 analog outputs: see http://fr.wikipedia.org/wiki/PWM) and 6 analog inputs 
converting 10 bits. In the most common version, the communication with the computer is via a USB port as shown in Figure 5. There are several versions of the Arduino, including a miniaturized version [12]. The card has an internal software system (editable) and user programs.

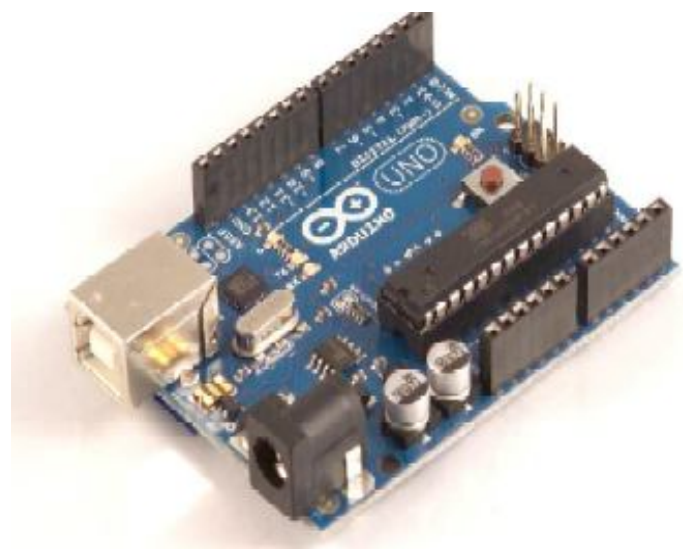

Figure 5. Arduino Uno board

Arduino programs are written in $\mathrm{C}$ or $\mathrm{C}++$. The Arduino IDE, as shown in Figure 6, comes with a software library called "Wiring" from the original Wiring project, which makes many common input/output operations much easier. Users only need define two functions to make a runnable cyclic executive program. The first function is setup (). This function is launched once at the start of a program that can initialize settings. The second function is loop (). It is called repeatedly until the board powers off.

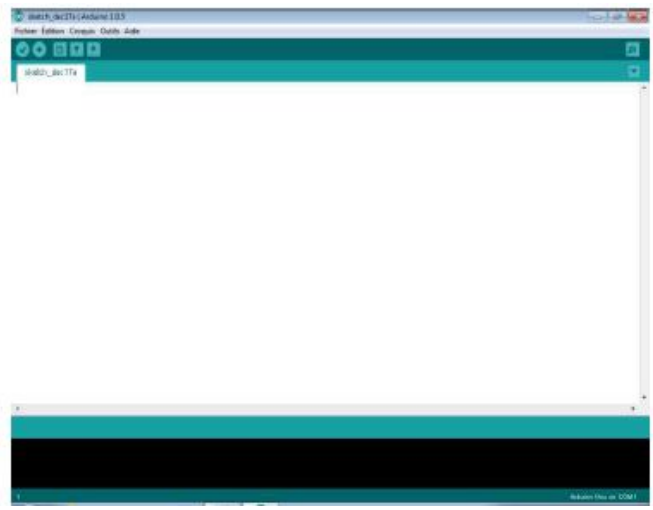

Figure 6. Arduino IDE

Our proposed diagnostic algorithm is defined as follows:

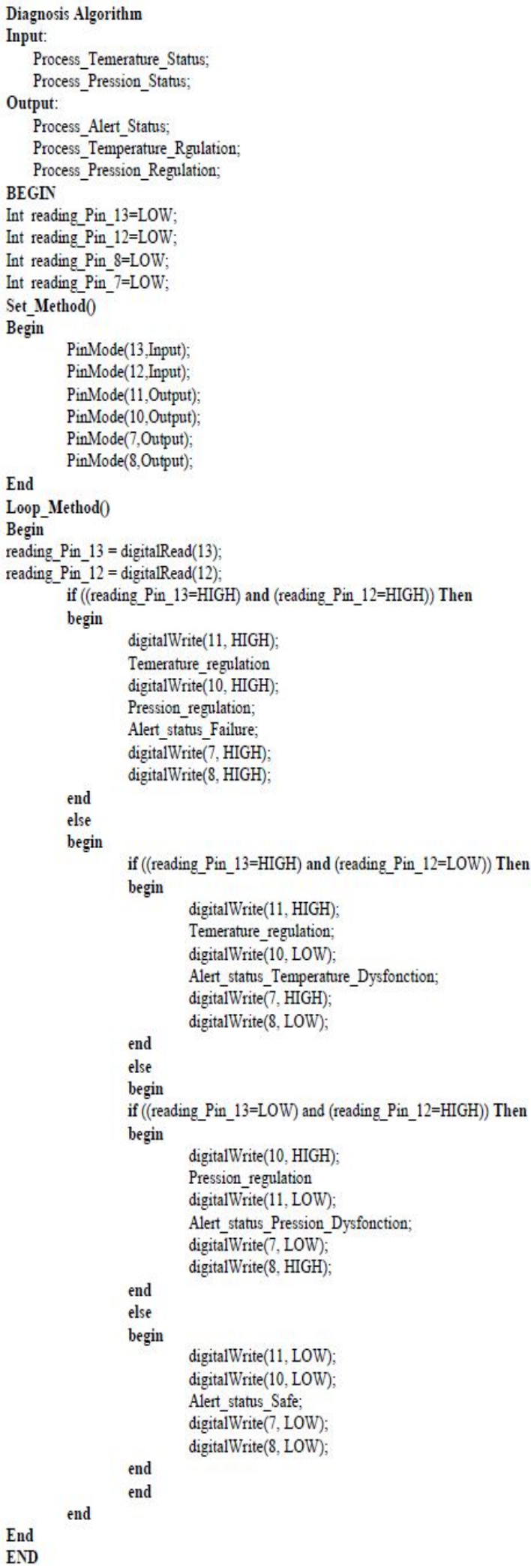

The program is written on the IDE Aduino then it was compiled. The executable code is loaded to the Arduino 
board. The system is connected to the Arduino. At this point, the Arduino board controls the system. The results of the implementation of our application are given as follows:

The system provides two output values. The first represents the state of the temperature and the other represents the state of the pressure, in which the operation of the system continues. Then the system generates these two values to the Arduino board, in the pin 13 we find the value indicating the status of the temperature, zero (0) means nothing to report, one (1) means that there is an anomaly to report. So, the state of pin 7 passes to state one (1). It triggers an alarm and proceeds by actuating the cooler. Pin 12 gives the value indicating the status of the pressure, zero (0) means nothing to report, one (1) means that there is an anomaly report. So, the pin 8 passes to state one (1). It triggers an alarm and proceeds by actuating the pressure regulator. In both cases the system switches to malfunction. In the event, the two anomalies occur together the system switches to state alarmed. In the worst case, we proceed by a forced shutdown of the system.

\section{CONCLUSION}

This paper has presented a brief introduction to the fields of FTC and FDI. An algorithm for monitoring a system was proposed by using Arduino technology.

As an emerging and active area of research in automatic control, fault-tolerant control has recently attracted more and more attention. When a fault occurs in a system, the main problem to be addressed is to raise an alarm, ideally diagnose what fault has occurred, and then decide how to deal with it. The problem of detecting a fault, finding the source/location and then taking appropriate action is the basis of fault tolerant control.

In our future work we are interested in neuro-fuzzy modeling and diagnosis.

\section{REFERENCES}

[1] L., Jiang.2011. Sensor fault detection and isolation using system dynamics identification techniques. PhD Thesis. University of Michigan, USA.

[2] M., Rodrigues, D. Theillol, M., Adama and D., Sauter. 2008. A fault detection and isolation scheme for industriel systems based on multiple operating model. Control Engineering Practice 16,2 (2008) 225-239.

[3] P., Rosa, C., Silvestre, J.S., Shamma, and M., Athans. 2010. Fault detection and isolation of an Aircraft using Set-Valued Observers. Georgia, USA.

[4] T., Escobet and L., Travé-Massuyès. 1995. Parameter estimation methods for fault detection and isolation. Campus de Terrassa, Barcelona, Spain.

[5] Y., Tharrault, G., Mourot, J., Ragot and D., Maquin. 2008. Fault detection and isolation with robust principal component analysis. Int.J.Appl.Math.Comput.Sci., 2008, Vol. $18, \mathrm{~N}^{\circ} 4,429-442$.

[6] M., Fliess, C., Join, and H., Sira-Ramirez. 2006. Closedloop fault-tolerant control for uncertain nonlinear systems. Ecole plytechnique. Palaiseau, France.

[7] Y., Wang, D., Zhou, S., Joe Qin, and H., Wang. 2008. Active fault-tolerant control for a class of nonlinear systems with sensor faults. International journal of control, automation, and systems, vol.6 n .3 , (2008), pp. 339-350.

[8] Y., Diao, K.M., Passino. 2002. Intelligent fault-tolerant control using adaptive and learning methods. Control Engineering Practice 10 (2002) 801-817.

[9] J., Stroustrup, and V.,D., Blonded. 2004. A simultaneous stabilization approach to (passive) fault tolerant control. Department of control engineering institute of electronic systems, Denmark.

[10] M., Staroswiecki, M. Blanke, and N., Eva. 2001. Concepts and methods in fault-tolerant control. Book.

[11] M., Staroswiecki, M., Blank, M., Kinnaert, J., Lunze. 2006. Diagnosis and fault-tolerant control. Springer.

[12] J.,N., Montagné. 2006. Initiation à la mise en œuvre matérielle et logicielle d'Arduino. Livret en français, Centre de Ressources Art Sensitif. 